\title{
A growing hepatic mass in a cryptogenic cirrhosis patient
}

\author{
Ji Soo Song ${ }^{1}$, Hee Chul Yu ${ }^{2}$, and Woo Sung Moon 3
}

Departments of ${ }^{1}$ Radiology, ${ }^{2}$ Surgery, and ${ }^{3}$ Pathology, Chonbuk National University Medical School and Research Institute of Clinical Medicine of Chonbuk National University Hospital, and Research Institute for Endocrine Sciences, Jeonju, Korea
A 23-year-old male patient who had been diagnosed with cryptogenic cirrhosis 30 months earlier presented with a tumorous lesion in the liver that increased in size from 4 to $11 \mathrm{~mm}$ in the past 15 months. Contrast-enhanced computed tomography (CT) demonstrated a $1.1 \mathrm{~cm}$-sized nodule in segment 4 of the liver with arterial hyperenhance-
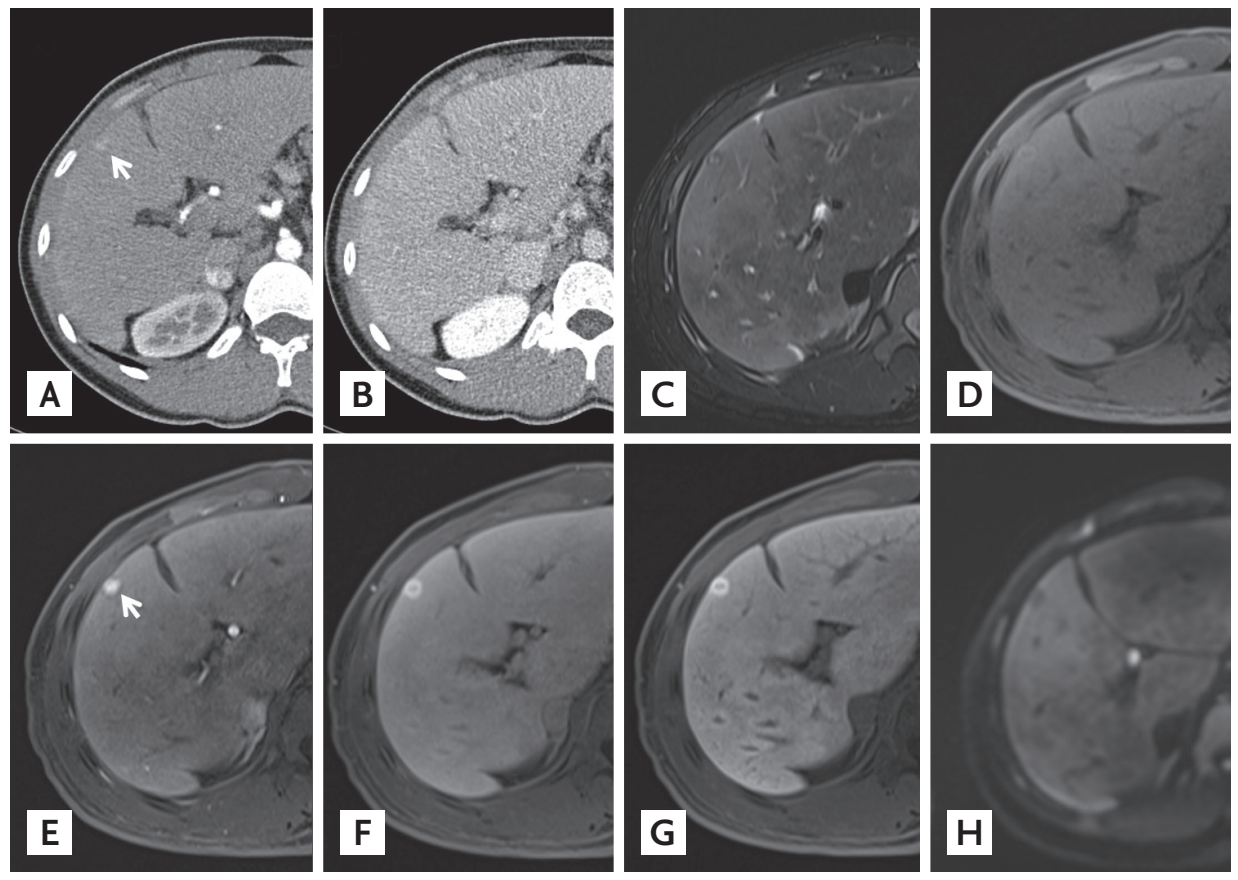

Figure 1. Contrast-enhanced computed tomography (CT) shows a $1.1 \mathrm{~cm}$-sized nodule in segment 4 of the liver (arrow) with (A) arterial phase and (B) delayed phase washout. (C) The nodule shows hypointensity with hyperintense central area on a T2-weighted fat suppressed image, and (D) slight hyperintensity on a T1-weighted fat suppressed image. (E) After the injection of gadoxetic acid, the nodule is homogeneously hyperenhanced on the arterial phase (arrow) with (F) hyperintensity on the transitional phase image. (G) On the 2o-minute hepatobiliary phase image, the nodule is hyperintense with central area showing hypointensity ("ethoxybenzyl [EOB] scar"). (H) There are no areas of diffusion restriction on diffusion-weighted image. 

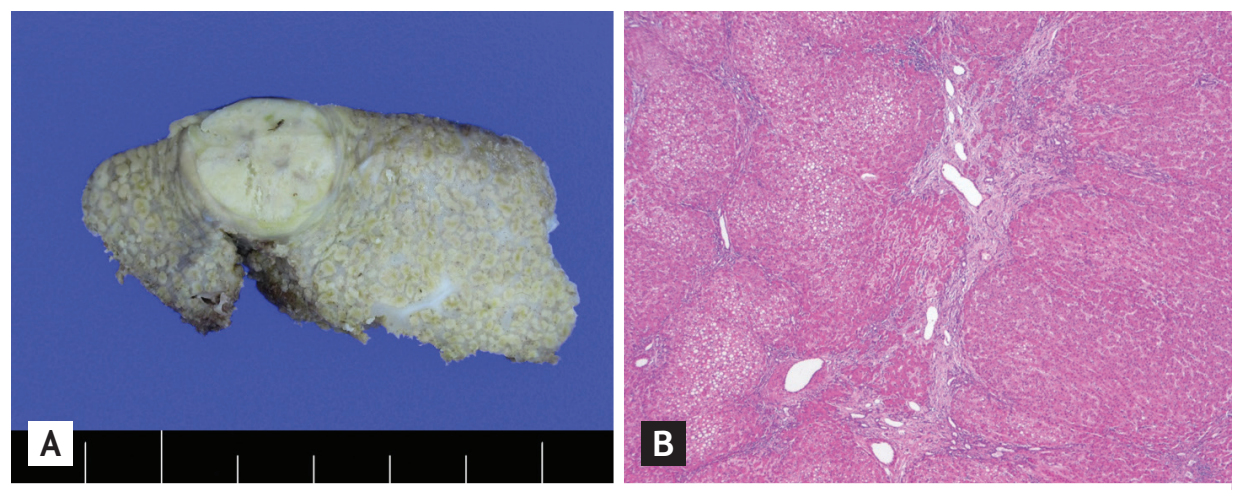

Figure 2. (A) The resected tumor is an encapsulated hyperplastic nodule and has central scar-like fibrosis containing anomalous vessels. (B) The lesion is composed of benign hepatic nodules arranged
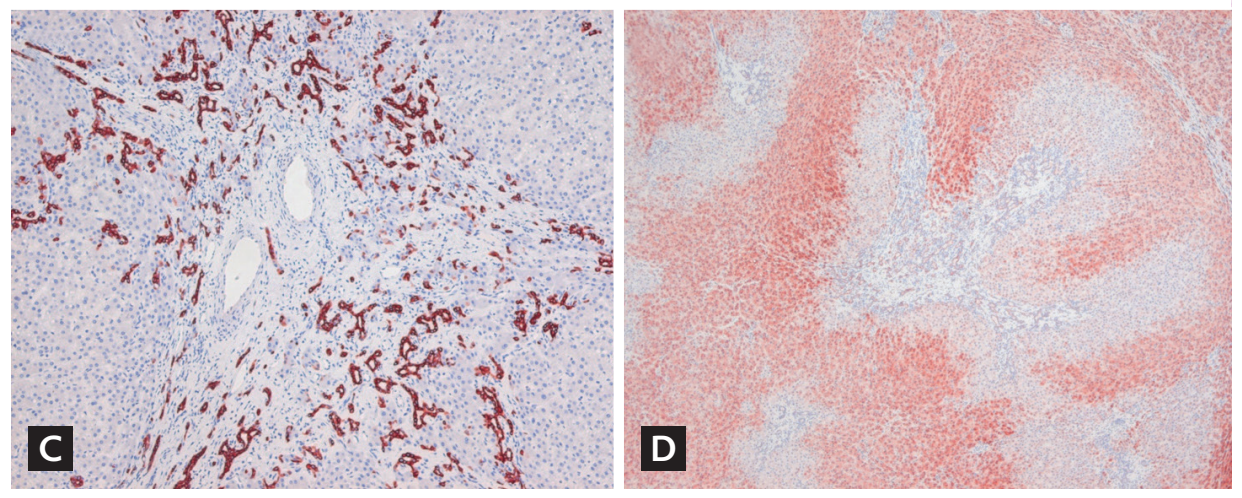
in plates, no more than two cells thick. The central scar contains portal tract-like structures with anomalous vessels and ductular proliferation (H\&E, ×40). (C) Immunostaining for keratin 19 highlights a prominent ductular reaction at the stromal-parenchymal interface $(\times 40)$. (D) Immunostaining for glutamine synthetase reveals a characteristic broad, anastomosing "map-like" pattern of staining (x40).

acid, strong homogeneous arterial enhancement without washout appearance (Fig. $1 \mathrm{E}$ ) and hyperintensity on the transitional phase image were present (Fig. 1F). On the 20-minute hepatobiliary phase (HBP) image, the nodule showed hyperintensity with central hypointensity (Fig. $1 \mathrm{G}$ ). There were no areas of diffusion restriction on a diffusion-weighted image $\left(b=800 \mathrm{~s} / \mathrm{mm}^{2}\right)$ (Fig. $\left.1 \mathrm{H}\right)$. The lesion was pathologically diagnosed as a focal nodular hyperplasia (FNH)-like nodule arising from cryptogenic cirrhosis (Fig. 2).

A FNH-like nodule is a benign hyperplastic nodule with a central scar arising from a cirrhotic liver. There is growing evidence that the image of gadoxetic acid-enhanced MRI can distinguish FNH or FNH-like nodules from other solid hypervascular tumors including HCC.
Absence of washout appearance on the portal venous or transitional phase, isointensity on diffusion-weighted imaging, presence of "ethoxybenzyl (EOB) scar," and homogeneous hyperintensity on the HBP image are characteristic features of FNH-like nodules on gadoxetic acid-enhanced MRI as shown in our case. Our case demonstrates that even though the initial CT scan of a nodular lesion in cirrhosis might show typical features of HCC, it would be beneficial to confirm the diagnosis by performing gadoxetic acid-enhanced MRI which might alternatively reveal the features of FNH-like nodules.

\section{Conflict of interest}

No potential conflict of interest relevant to this article was reported. 